\title{
Synopsis of Selected Discussion
}

The workshop proceedings were tape-recorded. This review focuses on formal and informal presentations not covered by papers in this collection. Thus, I have included summaries of the remarks by Ranck, Winson, Best, and Dalrymple, as well as snippets of discussion most germane to the goals of the meeting.

John O'Keefe's opening presentation brought out a number of questions and issues which would be recurrent themes throughout: What are the operational distinctions between spatial tasks and guidance tasks? What are the cues for spatial mapping? Would it not be possible to understand impaired performance on spatial tasks by animals with hippocampal damage in other terms, for example, differential task difficulty?

James Ranck recounted his observations of electrophysiological responses from single cells in rat hippocampus. Ranck has examined the responses of theta and complex-spike cells in situations with clear-cut spatial features such as the eight-arm radial maze and two-bar DRL operant tasks, as well as unlearned tasks such as pup retrieval in a "family" box. The spatial responses of cells differ in different situations, depending upon states of arousal or wakefulness.

Approximately $50 \%$ of cells have movementdirection correlates to their spatial response. Nevertheless, pure spatial responses can be observed independent of movement in the sense that a cell might respond in a given part of the test space even when the animal is passively transported to that place. In short, place cells in the hippocampus exist.

Ranck's informal remarks about the dependence of hippocampal unit responsiveness on the sleep-arousal dimension served as a useful introduction to remarks by Jonathan Winson on stimulation-elicited responses of hippocampal units in various stages of sleepwakefulness. Winson's summary of his observations follow:

\section{Behaviorally Dependent Gating of Neuronal \\ Transmission in the Hippocampus. Jonathan Winson}

Winson first noted that the findings he was about to present did not relate in any obvious manner to the role of the hippocampus in learning and memory but represented a heretofore unexplored aspect of hippocampal physiology which must ultimately be integrated into our overall view of the function of that structure.

Per Andersen and his colleagues first delineated the physiological relations within the hippocampal trisynaptic circuit consisting of the granule cells of the dentate gyrus, the CA 3 pyramidal cells, and the CA1 pyramidal cells of the hippocampus. Synaptic action at each stage of this circuit is excitatory, and, under appropriate conditions, a single pulse applied to the perforant pathway is transmitted through the entire trisynaptic chain. The Andersen experiments were performed in anesthetized preparations. However, there was reason to believe that the excitability of hippocampal cells, and hence the efficacy of neuronal transmission through the trisynaptic chain, might depend upon behavior. First, the hippocampal formation is known to receive projections from both locus coeruleus and raphe nuclei, and the firing rates of cells in these regions are reported to vary systematically with behavior. Hence, brainstem cells might exert behaviorally specific tonic influences on the excitability of hippocampal neurons. Second, theta rhythm occurs in some behaviors, such as exploration and REM sleep in the rat, but is absent in others. Theta rhythm is probably accompanied by cyclic changes in intracellular potentials which should be capable of affecting neuronal excitability. Based on these considerations, a study was undertaken several years ago by Winson and Abzug in which neuronal transmission through the trisynaptic chain was measured in freely moving rats. Experiments were performed during four behavioral states: slow-wave sleep (SWS), rapid-eye-movement (REM) sleep, the still-alert condition, and exploratory behavior. During each of the four behaviors, a single pulse of electrical stimulation was applied to the perforant pathway, and field responses were recorded at each juncture of the trisynaptic chain. In contrast to the unrestricted neuronal transmission that occurs in anesthetized animals, it was found, in freely moving animals, that at each stage of the trisynaptic circuit the signal originating in the perforant pathway was either transmitted freely or restricted in transmission according to the behavioral state of the animal. The net result was a selective transmission of the perforant path signal through the hippocampal circuitry to particular limbic target areas, the pattern of transmission being dependent on behavior. A major site of this behaviorally dependent modification of transmission was found to be the dentate gyrus with additional modulation occurring in CA1.

More recent experiments were undertaken to discover whether brainstem influences might, indeed, be responsible for the behavioral changes that occur in neuronal transmission through the hippocampal formation. Attention was restricted to the first stage of such transmission, that is, from the perforant pathway through the dentate gyrus and the two behavioral states, SWS and the still-alert condition. The paradigm used in these experiments was the same as in the previous study, with the exception that shortly before applying the test pulse to the perforant pathway a stimulus was applied to the site in the brainstem. Stimulation sites encompassed the entire pons and medulla, on the midline and mediolaterally. It had been found previously that neuronal transmission from the perforant pathway through the dentate gyrus was facilitated during SWS as compared with the still-alert state. Loci were found in the brainstem in which prestimulation (with thresholds on the order of $30 \mu \mathrm{A})$ resulted in a marked additional facilitation of 
neuronal transmission during SWS, while prestimulation in the still-alert state did not affect neuronal transmission. All of these loci were on the midline within the limits of the raphe nuclei. Stimulation of the median raphe nucleus was particularly effective. The minimum time between the application of a prestimulus to a raphe nucleus and the application of the perforant path test pulse at which facilitation could be elicited was $5 \mathrm{msec}$. It was further determined that lesions of median raphe nucleus eliminated the normal behavioral facilitation of neuronal transmission that occurred during SWS.

Taken together, these findings suggested that tonic influences arising in the raphe nuclei might be responsible for the facilitation of neuronal transmission observed in normal animals during SWS. Furthermore, there was apparently a potent suppressive influence operating in the hippocampus during the still-alert state since raphe prestimulation was ineffective in producing facilitation during this behavior.

Studies were under way to determine if the effects produced by raphe stimulation were mediated by serotonin and to identify the source of the suppressive influence present during still-alert behavior.

Phillip Best began his formal presentation by reviewing attempts to find electrophysiological correlates of classical conditioning in hippocampal units of rats. These experiments indicated that the response of a unit (typically a complex-spike cell) to the CS, whether with an increased or decreased rate of firing or no change, depended both on whether the CS had been preexposed, that is, made a latent inhibitor, and on whether the animal was asleep or awake.

Best then described experiments in which motion pictures of rats on an eight-arm radial maze were shown to undergraduate psychology students, who were asked to classify the response of hippocampal units (on the sound track) as an animal performed this task. Students had no difficulty classifying units as to their spatial reactivity: Of 31 units, 18 increased firing rate in only one arm, and 9 of these were "pure" spatial cells in the sense of having no obvious movement or direction component. Seven units were spatial but gave an "off" response instead of an "on" response. The remaining spatial units had wider receptive fields, increasing (or decreasing) their firing rate in two out of the eight arms. Only 5 units failed to show some form of spatial response. Best noted that most units were theta cells and that they tended to fire briefly at choice points (VTE-ing?) with "motion punctuation."

Best then described the effects of rotating the maze and lesioning the fornices or entorhinal areas. For nonlesioned animals, maze rotation did not change the spatial response characteristics of 17 out of 17 units. Thus, these units maintained their receptive field with respect to place (i.e., extramaze coordinates) rather than shifting their receptive field to the new position of the arm(s) that had elicited a response prior to rotation of the maze.
Best felt that this observation ruled out the possibility that the original receptive fields could be accounted for in terms of subtle intramaze cues, for example, differential reverberations from the floor of the apparatus.

For fornix-lesioned animals, firing rates increased in places beyond the original receptive field, and in some cases receptive fields followed the new position of the arm rather than remaining at the original place. Of 24 spatial units, however, 11 showed spatial persistence despite the overall higher firing rate out of the original field. Best attributed this spatial persistence to the fact that the lesions were comparatively small. By contrast, the receptive fields of the 15 units that survived lesioning of entorhinal areas either failed to maintain their spatial receptive field or else spatial specificity of response was obscured by an overall increase in firing rate out of the original field. In general, therefore, not only did lesions of hippocampal inputs produce the typical deterioration of performance on the eight-arm radial maze, but also this performance deficit was related to a decrease of one kind or another in the spatial "tuning" of hippocampal units.

The question was raised as to whether spatial units are defined as soon as the animal is placed in the apparatus or whether their spatial tuning evolves with experience. A. J. Hill felt that a majority of spatial units in the hippocampus express their tuning characteristics immediately and that a "learning curve"' is not generally evident.

Andrew Dalrymple, a doctoral student at McMaster University, reported on his and Abe Black's thinking on how to characterize the consequence for learning and memory of brain dysfunction, particularly when the dysfunction involves the hippocampal formation.

Dalrymple reviewed the classic controversy between S-R behaviorists and the cognitive map position of Tolman. He suggested that tasks that emphasize the animal's need to "reason" and adopt strategies are those most relevant for relating the effects of brain dysfunction to psychological processes. Such tasks include many traditional latent learning and memory tasks such as the Maier three-table problem and the Tolman-Gleitman E-maze.

A normal animal's performance in such tasks depends on a number of possible strategies (including place and response) which, in turn, depend on associations between and among all sorts of events, including cues, responses, and reinforcers. The experimenter's task is to identify the animal's strategy in the task and the components of the task which determine that strategy.

Thus, the psychological consequences of brain dysfunction should be approached within a general theory of what happens to the normal animal in the three phases of the prototypical "reasoning" experiment: In Stage 1 of such an experiment, there 
is established an organized set of central representations which includes a cognitive map. In Stage 2, a subset (component) of this central representation is modified by the introduction of the reinforcer, that is, by a conditioning procedure. In Stage 3, the relevant components are reactivated or recalled and behavior is modified, and "action at a distance" is possible. That is, what the animal chooses to do in one place depends upon what happened at another place.

After presenting some experimental evidence to illustrate the importance of strategy and component identification within the three stages of reasoning experiments, Dalrymple closed by citing Tolman's list of factors that potentially influence strategy selection by yielding "narrow-strip" cognitive maps. This list includes stimulus-poor environments, strong motivation, overtraining, and brain lesions.

David Olton and two of his collaborators, James Becker and Gail Handelmann, described experimental evidence related to their hypothesis that the hippocampus is involved in working memory. Their data support the idea that reference memory, which could have both spatial and guidance components, is not affected by hippocampal dysfunction so long as working memory is not a crucial aspect of the task. Controversy centered on whether a given test situation would satisfy everyone's definition of whether or not it successfully emphasized the distinction between working and reference memory, on the one hand, and spatial and guidance, on the other. This turned out to be a recurrent theme, and as the workshop proceded, data and potentially useful experiments bearing on this question were introduced. Nevertheless, it proved extremely difficult for proponents of the spatial mapping theory to agree with Olton's group on the strategies and components of memory used by rats in any given task.

A portion of the discussion elicited by Olton's group dealt with the use of kainic acid to make selective fiber-sparing lesions (reported by Gail Handelmann). This concerned technical matters related to spread and incidence of seizures. Jarrard has also used this technique in connection with his studies of the role of components of the hippocampal formation in learning and memory.

O'Keefe asked whether working memory was more prone to interference than reference memory and, if so, whether this did not imply that hippocampectomized animals, presumably without working memory, would be less, rather than more, prone to interference. Nadel cited evidence that the eight-arm radial maze is not particularly subject to interference (Maki, W. S., Brokofsky, S., \& Berg, B. Spatial memory in rats: Resistance to retroactive interference. Animal Learning \& Behavior, 1979, 7, 25-30) and that this suggests that working memory, that is, an interference-prone limited-capacity memory, is not involved in this task.
Jarrard mentioned experiments with a 12-arm radial maze on which only 4 arms were baited. Retroactive interference procedures between original learning and the test impaired performance in this task relative to controls, suggesting disruption of working memory. However, hippocampal damage impaired test performance for both interference and control animals. Nadel suggested that these experiments were consistent with the spatial mapping hypothesis because without a functioning hippocampus animals not normally prone to interference, because they can employ place strategies, fall back on interference-prone cue strategies.

Nadel described experiments designed to clarify the role of the hippocampus in working memory. One experiment was similar to Jarrard's in that it employed a radial maze with only a portion of the arms baited. In this case, the apparatus was an eight-arm maze, and only four arms were baited, each with a different number of food pellets. Instead of introducing interference procedures, Nadel counted two types of errors: A working-memory error occurred when the animal reentered a just-visited baited arm; a reference-memory error occurred when the animal entered an arm that had never been baited. According to Nadel, animals with hippocampal damage showed a higher incidence of both sorts of errors than did normal animals.

Nadel mentioned another experiment employing 4 baited arms out of 8 of a radial maze. In one case, animals were presumably forced to employ a strategy based on intramaze cues because the extramaze context was too impoverished to support place learning. Animals with hippocampal damage were more impaired on the test than controls when a cue-rich extramaze context was employed, presumably because the lesioned animals could no longer employ a place strategy. As before, lesions seemed to increase the incidence of both reference-memory and workingmemory errors. However, with the impoverished context, in which place strategies were presumably unavailable in the first place, lesioned animals did not differ from normals.

The apparent conclusion to be drawn is that the distinction between place and cue learning discriminated lesioned animals from normals, but the distinction between working and reference memory did not.

Paul Ellen asked how one could know what strategy the animal employs from trial to trial. Olton suggested that, in order to infer what stimuli the animal employs, one must manipulate stimuli. He went on to offer some operational definitions of working memory and reference memory: Working memory contains information useful for one trial but not for subsequent trials. Reference memory contains information useful for more than one trial. Olton went on to suggest that working memory might also be characterized as subject to within-trial interference. O'Keefe questioned 
whether susceptibility to interference should be part of an operational distinction between working and reference memory.

Jarrard, O'Keefe, Olton, and others cited tasks with both working- and reference-memory components (e.g., single alternation, two-way shuttle avoidance), and a consensus developed that useful experiments would employ independent variables that would unambiguously pit place and cue learning against working and reference memory in a 2 by 2 (orthogonal) experimental design with lesions and controls as the third orthogonal factor. Precisely which tasks would best lend themselves to this approach remained in doubt.

In sum, the working memory hypothesis seemed attractive, but some experimental evidence formally presented (e.g., Jarrard) or mentioned in passing (e.g., Nadel, Hirsh) appeared inconsistent with this hypothesis. This reporter was impressed by the difficulty of attributing errors to dysfunction of working memory, on the one hand, and reference memory, on the other. The problems of component and strategy identification discussed by Dalrymple were very much a matter of concern.

Before the presentations on the role of the hippocampus in classical conditioning of the rabbit's nictitating membrane response, O'Keefe described preliminary electrophysiological evidence that some cells in the hippocampus of rabbits respond selectively to place: Of 42 cells in three rabbits tested in an "openfield" context, 24 were place cells, 12 were theta cells, and 6 were unclassified. O'Keefe's impression was that there is a greater proportion of theta cells in CA1 of rabbits than of rats. O'Keefe went on to describe, off the record, some of his own observations of the electrophysiological responses of hippocampal units during classical eyeblink conditioning.

Nadel's formal presentation attempted to relate evidence of hippocampal involvement in classical conditioning situations to contextual (place) stimulus control: In situations in which contextual control plays a prominent role, for example, in certain conditioned inhibition situations in which the occurrence of the unconditioned stimulus (US) is negatively correlated with the nominal (as opposed to contextual) conditioned stimulus (CS), hippocampal damage should impair performance. When contextual (place) cues do not play a part, for example, in certain USpreexposure experiments, lesioned animals should perform in the same way as normal animals.

In essence, then, Nadel suggested that whatever distinguishes an animal with hippocampal damage from a normal animal in classical conditioning experiments might be interpreted as failure by lesioned animals to recognize the place where the experimental contingencies unfold. Richard Thompson was not alone in questioning this particular extension of the cognitive map theory of hippocampal function.
Nadel asked what the hippocampal electrophysiological correlates of classical conditioning described by Thompson and Berger had to do with the evidence from Solomon and Moore that the hippocampus is involved in tuning out irrelevant events. To him, it seemed paradoxical that hippocampal units do not respond during control procedures in which the CS and US are explicitly unpaired. Yet, this would appear to be a situation in which the hippocampus would be tuning out the CS. Solomon and others were reluctant to admit a paradox.

The last session of the workshop was devoted to discussion of issues raised in connection with earlier formal and informal presentations. The initial discussion at this time concerned the question of dissociating working and reference memory from place and cue learning. This discussion has been summarized above.

Solomon asked for definitions of the term "context" and how this concept might be related to space and time.

Ted Berger introduced the idea of temporal context as defined through repetition of events in a fixed sequence. Thus, the CS in classical conditioning can be viewed as part of the context in which the US occurs, even though it need not be present at the instant that the US occurs, for example, as in a trace conditioning procedure.

Moore suggested that the distinction between foreground or proximal stimuli and background or distal stimuli might be one useful dimension for defining what is meant by context.

Hirsh felt that "temporal schema" must be part of cognitive map. Thus, time is but one "setting factor," as illustrated by one's differing reaction to the suggestion of a cocktail at 9 a.m. and to a similar suggestion at 6 p.m.

Olton asked whether there was a fundamental distinction between spatial and temporal maps. Many felt that they could not be separated. For example, it was suggested that if behavior can be described in a sequence of programmed steps, then time and space cannot (indeed should not) be separated.

Moore believed that defining a temporal context or map was a doubtful enterprise from a "real-time" perspective. Learning theories traditionally attempt to describe the development of associative networks or cognitive structures as a function of time or experience. Thus, whereas a spatial representation might remain invariant over time, temporal invariance seems to imply that time can stand still.

Solomon asked how one could change a temporal context. Berger suggested that this might be done by altering the sequence of events or by introducing into or subtracting events from this sequence. Solomon then asked Nadel to account for data suggesting that rabbits with hippocampal ablations were more prone to external inhibition than were normals: Did this para- 
digm involve spatial mapping or contextual stimulus control?

Olton asked whether classically conditioned responses could "track" an alternating schedule of reinforced trials. A few such instances were described, and Jarrard recalled a report that cardiac-conditioned rats with hippocampal damage do not track an alternating schedule of trials as well as normals.

Solomon proposed long-trace conditioning paradigms as relevant to Nadel's proposal that disruption of control over conditioned responding by contextual stimuli lies behind differences between animals with hippocampal damage and normals in classical conditioning situations. Hippocampal animals, unable to maintain a "focus" (term suggested by Olton) on the conditioning context, might be expected to be deficient in situations in which the CS is introduced and then withdrawn before the occurrence of the US.

Solomon then asked Nadel to account for data suggesting that conditioned inhibition is not disrupted in animals with hippocampal damage. Nadel replied that this disruption would be expected only in conditioned inhibition situations in which the context provided the excitatory support for inhibition demanded by the Rescorla-Wagner model. No one could recall a relevant experiment that included both summation and retardation tests of conditioned inhibition.

Participants and guests were given the opportunity to get off a "parting shot" or thought:

Jarrard urged greater precision in specifying the location and extent of lesions. He also urged greater attention to the role of subicular efferents as opposed to the traditional focus on the fimbria-fornix system.
Kesner speculated that the hippocampus was involved whenever order or conditionality was a prominent aspect of behavior.

Salafia expressed the view that the evidence offered for hippocampal involvement in spatial and temporal domains was not really in conflict.

Ranck noted the appeal of the hippocampal system as a venue for reductionistic or cellular approaches to behavior, but he warned against mistakes of "microphrenology," that is, the structure must always be viewed as part of a multifunctioned system.

Dalrymple suggested that conditioned discrimination problems based on circadian rhythms would be useful additions to the literature on hippocampal function. It was also suggested that the number of tasks and species employed in research on hippocampal function should be increased.

Olton reiterated the need for consensus on the experimental designs and tasks that would yield unambiguous evidence regarding the various theories of hippocampus function.

Hirsh expressed the view that three theories-tuning out irrelevant events, working memory, and spatial mapping-overlapped to such an extent that new alternative conceptualization which achieve a greater degree of generality are worth pursuing.

Jeff Willner concluded by urging greater sophistication in analyzing the behavior of normal animals, both in terms of their natural histories and in terms of the behavioral theories most relevant to the task at hand. Such sophistication is essential for continued progress in behavioral brain research. 